\title{
Clinical value of the cortisol secretion rate
}

\author{
C. L. COPE AND J. PEARSON \\ From the Postgraduate Medical School of London
}

SYNOPSIS The isotopic estimation of the cortisol secretion rate in man is now an accepted and reliable method of assaying adrenal cortical function.

Seven years' experience with its clinical use is reviewed and some practical aspects of technique are considered.

The mean normal resting cortisol secretion rate is $16.2 \pm 5.7 \mathrm{mg}$. daily. In all of 14 cases of hypopituitarism studied secretion has been less than $2 \cdot 1 \mathrm{mg}$. daily. In all of 26 cases of establishedo Cushing's syndrome, the cortisol secretion was above $36 \mathrm{mg}$.

The method can be used to follow day-by-day changes in adrenal cortisol activity and examples. of such use are given.

The urinary 17-ketogenic steroid excretion frequently gives results which conflict with secretion? rate estimates, and can lead to erroneous clinical conclusions.

Understanding of adrenal cortical metabolism has advanced with great speed during the last 10 years, and techniques previously difficult are now becoming more generally feasible for clinical purposes. With the increasing availability of apparatus for the quick counting of the isotopes of carbon and of tritium, and with the recent introduction of commercially available tritium-labelled steroids at a modest price, it is now possible to introduce methods for measuring the secretion rates of cortisol and of some other similar steroids into semi-routine use not only in research studies, but also in the investigation of difficult or ambiguous cases of adrenal disorder.

Nearly all the pioneer work on suitable techniques using isotopes was carried out with ${ }^{14} \mathrm{C}$-labelled steroids. But although these are now commercially available their cost is high. Tritium-labelled corticosteroids are, however, so very much cheaper that the cost for a single test need not now exceed a few pence.

It is our purpose to review seven years' clinical experience with cortisol secretion rate determinations, during the last three of which tritium-labelled steroids have been widely used. During this time several hundred secretion rates have been measured, the results frequently proving of major clinical value. The most convenient method, and the one we have used, has been that originally introduced by Cope and Black (1958a), though a number of modifications have been introduced at times for special purposes.

Received for publication 2 April 1964.
The principle of the method is to introduce into

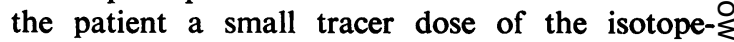
labelled steroid and to collect all urine passed during the next 24 or 48 hours. A known fraction of this urine collection is then hydrolysed with the enzyme $\beta$-glucuronidase in order to liberate the steroid metabolites and these are then extracted with윽 chloroform. The main metabolites, tetrahydro- $\frac{3}{5}$ cortisone and tetrahydrocortisol, are separated from the crude chloroform extract by paper chromatography and elution of the appropriate areas fromo the paper. The specific activity of these steroid metabolites, that is, their isotope content, is then found by determining the steroid content of part of 0 the eluate by any suitable method, and measuring the isotope content of another portion of the same응 eluate. The steroid secretion rate is usually calculated simply as the dose of isotope administered divided by을 the specific activity of the isotope isolated, both being expressed in the same units. In such a test onlyo the measurement of the dose given and the determin- $N$ ation of the specific activity of the steroid in the final eluate need be quantitative.

PRACTICAL AND TECHNICAL CONSIDERATIONS

THE RADIATION HAZARD The radiation exposure arising from such a test is believed to be almost negligible and is $\vec{O}$ certainly far less than that resulting from a single $\overrightarrow{\mathbb{D}}$ diagnostic dose of radio-iodine. The radiation exposure to gonads resulting from a diagnostic dose of $1 \mu \mathrm{c}$. of $\varrho$ ${ }^{14} \mathrm{C}$ labelled cortisol has been calculated as $0.006 \mathrm{mrad}$. 
(Cope and Black, 1959a). This corresponds to the radiation received by each member of the population from natural sources in about half an hour. The radiation exposure when tritiated cortisol is used will be considerably less than this and seems therefore to be entirely negligible.

STABILITY OF TRITIUM LABEL The firm attachment of the isotope to the steroid molecule during all the stages of metabolism within the body is an essential requisite of steroid secretion rate estimations. Whereas a ${ }^{14}$ carbon label is firmly embedded in the carbon skeleton of the steroid nucleus, hydrogen and tritium atoms are only attached to this ring and are theoretically vulnerable to detachment. It is necessary, therefore, to be sure that no appreciable portion of the tritium becomes detached from the molecule during the processes of metabolism. This is best done by comparing the behaviour of the tritiumlabelled cortisol with that of the stable ${ }^{14} \mathrm{C}$-labelled cortisol. Since metabolic conditions in any individual may vary from day to day it is desirable to test both steroids simultaneously, and this means that each isotope must be measured in the presence of the other. We have made this comparison in a series of five subjects. Doses of both ${ }^{14} \mathrm{C}$-labelled and tritium-labelled cortisol were given simultaneously by mouth, and the ratio of ${ }^{14} \mathrm{C}$ to tritium in the administered dose was compared with the ratio of these two isotopes found in the tetrahydrocortisone and tetrahydrocortisol subsequently recovered from the urine. The ${ }^{41} \mathrm{C} /{ }^{3} \mathrm{H}$ ratio for the five administered doses was 1.49 , and the mean ratio in the recovered metabolites was $1 \cdot 51$, a $2 \%$ difference which is within experimental error. In no single case did the ratio of the isotopes in the urinary metabolites vary more than $5 \%$ from that of the administered dose. This means that both the tritium-labelled and the ${ }^{14} \mathrm{C}$-labelled cortisol will give the same result for the secretion rate, and no appreciable amount of tritium label can have been lost during the process of metabolism and excretion of the dose from the body.

MODE OF ADMINISTRATION OF LABELLED CORTISOL In the original work on cortisol secretion rates Cope and Black (1958a) suggested that the dose could be given by mouth. This is not only more convenient than the intravenous route but leads to the dose entering the blood stream more slowly, a desirable feature. Two theoretical objections to the oral route can, however, be raised: (1) absorption from the alimentary tract may be incomplete and (2) the absorbed dose which has to pass through the liver may have a different metabolic behaviour from that of endogenous cortisol which enters the systemic circulation directly. In an effort to meet these possible criticisms, Cope and Black (1958a) compared the two routes in five subjects, but, as only one isotope was available at that time, the two routes had to be tested on different days and spontaneous fluctuations in activity in the individual could not be excluded. Now that both tritium-labelled and ${ }^{14} \mathrm{C}$-labelled cortisol are available it is possible to administer doses by both routes simultaneously in the same person and to follow the behaviour of each without interference from the other. We have made this simultaneous comparison between oral and intravenous routes in six subjects, of whom five had normal adrenal cortical function and one had Cushing's syndrome. In three cases tritium-labelled cortisol was given orally, and in the other three it was given intravenously. In each case ${ }^{14} \mathrm{C}$-cortisol was given simultaneously by the alternative route. Simultaneous measurements of cortisol secretion rate could thus be made with both isotopes and by both routes of administration. The results in the six subjects (Table I) may be summarized as follows. The mean secretion rate by the oral route was $23.3 \mathrm{mg}$. daily and by the intravenous route $24 \cdot 3 \mathrm{mg}$. daily; the estimated rate by ${ }^{14} \mathrm{C}$ cortisol was $23.0 \mathrm{mg}$. daily and by ${ }^{3} \mathrm{H}$ cortisol $24.6 \mathrm{mg}$. daily. These results may be regarded as agreeing within the limits of experimental error. It is clear, therefore, that under ordinary clinical circumstances little is gained by giving the dose intravenously. The latter route may be used, however, when special accuracy is required, or when alimentary absorption is expected to be incomplete.

TABLE I

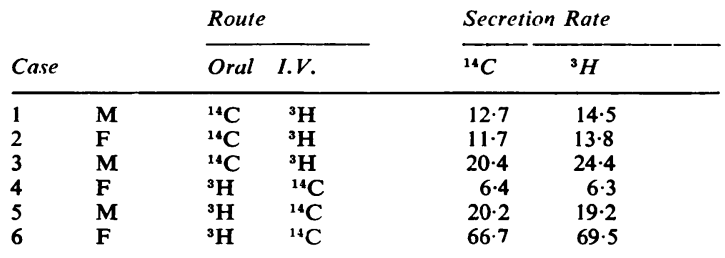

SEPARATION OF THE CORTISOL METABOLITE The reliability of the secretion rate estimation depends on the separation of the cortisol metabolite from the urine in a state of purity sufficient for an accurate measurement of the specific activity to be made. In clinical practice it is generally necessary to make a compromise with high accuracy, since its pursuit greatly increases the technical work involved. We have chosen the toluene/propylene glycol chromatographic system for the separation of the metabolite because it is the simplest system to use. It requires no accurate temperature control nor careful equilibration, and a single paper can carry a considerable weight of extract without overloading, although a single chromatographic separation carries the risk of insufficient purification. The main source of contamination is likely to be from cortolones which are also cortisol metabolites but which are not estimated by the usual steroid reagents although they will contain tritium. Their presence will lead to an estimate of the secretion rate which is rather too low. The tetrahydrocortisone and tetrahydrocortisol can be further purified by running again on a second paper in either the Bush $B_{5}$ (Bush, 1952), or the $E_{2} B$ system (Eberlein and Bongiovanni, 1955) but the extra work involved will rarely be justified.

In a well-developed chromatogram in the toluene/ propylene glycol system such contamination will affect the tetrahydrocortisol but not the tertahydrocortisone. We have compared the specific activities of these two metabolites separated by a single chromatograph in a consecutive series of 61 cases. In this the average difference between the two specific activities was $15 \%$. In one third 
of the comparisons the difference was less than $10 \%$, and only in seven instances did it exceed $30 \%$.

In practice it will usually suffice to elute the tetrahydrocortisone and the tetrahydrocortisol together and to determine the specific activity of the mixture. If this is done the error due to contamination should be less than $15 \%$ in more than $90 \%$ of the cases. Discrepancies of similar degree between specific activities of these two major metabolites seem to have been found also by Van der Straeten, Vermeulen, and Orie (1963). For general clinical diagnostic purposes such errors in measuring the secretion rate are relatively unimportant since they are likely to be less than the day-to-day variation of adrenal activity in the individual. Further chromatography, carried out in an effort to obtain greater precision, will not always lead to close approximation of the two specific activities. In estimations of the secretion rate it is not usually logical to do this unless checks on the efficiency of elimination of tritium in the urine are also carried out, because variations in the latter can introduce even larger errors if not detected and allowed for.

CALCUlation of THE SECretion RATE The usual method of calculation is to regard the secretion rate as indicated by the dose given divided by the specific activity of the metabolite obtained from the urine, and this will be valid if the excretion of the dose has been complete. But it not infrequently happens in clinical practice that renal function is impaired, and in such circumstances excretion of the dose of isotope will be incomplete. This will not usually introduce a serious error when the blood urea is below $50 \mathrm{mg}$. per $100 \mathrm{ml}$. But the higher the blood urea rises above this level, the greater the error likely to be introduced, until with blood urea levels above $200 \mathrm{mg}$. per $100 \mathrm{ml}$. the cortisol secretion rate estimates will often be two or even three times the true figure.

In such situations estimates of secretion rate can only be obtained if the actual tritium content of the urine is also estimated and appropriate corrections are made. The manner of correcting for poor elimination has been discussed by Cope and Pearson (1963). Although applied in that paper to aldosterone, the principle seems to be equally applicable to cortisol.

\section{RESULTS}

NORMAL RANGE OF CORTISOL SECRETION In a recent consecutive series of 25 convalescent hospital patients of both sexes at rest, and having no known reason for a raised adrenal cortical activity, the cortisol secretion rate has varied from $6.3 \mathrm{mg}$. daily to $28.6 \mathrm{mg}$. daily, the mean figure being $16.2 \pm 5.7$. Slightly higher figures are found in men than in women.

HYPOPITUITARISM In panhypopituitarism very low cortisol secretion rates have always been found and there has been no overlap with the normal range. In 14 cases of clinically recognizable panhypopituitarism the cortisol secretion rate has been between 0.6 and $2.1 \mathrm{mg}$. daily in all (mean $1.4 \mathrm{mg}$.). We have come to regard a cortisol secretion rate of above $2.5 \mathrm{mg}$. as good evidence that some pituitary adrenocorticotrophic function is persisting. ADDISON's DISEASE The findings in hypopituitarism은 stand in contrast to those in Addison's disease in $\overline{\bar{\alpha}}$ which cortisol secretion rates have ranged between $0.6 \mathrm{mg}$. and $19 \mathrm{mg}$. per day, thus overlapping considerably the normal range.

HYPERADRENALISM In Cushing's syndrome the range of secretion rates likely to be encountered seems to be dependent on the aetiology. In a con-ำ secutive series of 26 cases of Cushing's syndrome, all! of which were shown to be corticotrophin-dependent $\vec{\circ}$ and which were believed to be due, therefore, tocortical hyperplasia, the cortisol secretion rate varied between 36 and $138 \mathrm{mg}$. daily, the mean for음 the whole group being $64.3 \mathrm{mg}$. daily (Fig. 1), avalue four times the normal mean.

Much higher secretion rates are commonly found in subjects with carcinoma of the adrenal. Three such cases which were studied had cortisol secretion rates of 137,211 , and $316 \mathrm{mg}$. a day respectively. $\mathscr{\circ}$ The mean for this small group is thus $221 \mathrm{mg}$. a day. But an even higher figure was found in a patient suffering from severe hypercorticism due to an oat-cell carcinoma of the bronchus. In this man $418 \mathrm{mg}$. of cortisol was being produced daily. The highest secretion rate we have so far recorded was in $\frac{\mathbb{Q}}{\square}$ a patient with Cushing's syndrome due to adrenal $\vec{F}$ hyperplasia who had received corticotrophin, the $\frac{0}{3}$ adrenal thereby being stimulated to produce $544 \mathrm{mg} . \bar{J}$ of cortisol per day. But there is no reason to believe that even this figure is the highest attainable.

But although the established cases of Cushing's syndrome due to hyperplasia have all had cortisol secretion rates above the upper limit of the normal. range, it is clear that ambiguous or borderline cases $\delta$ will sooner or later be encountered, and indeed several have been seen. Some of these appear to be윽 in the early stages of development of a full Cushing's

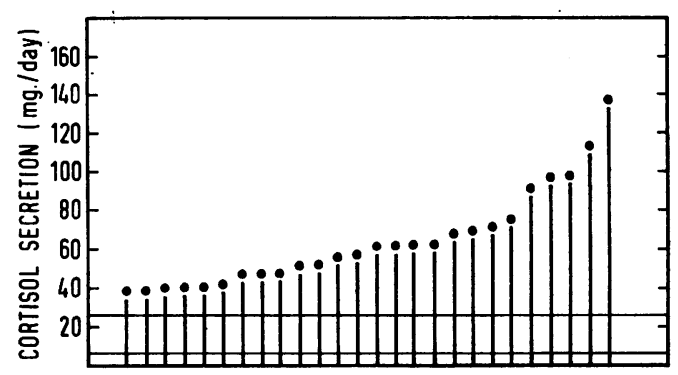

FIG. 1. Cortisol secretion rates in a series of 26 cases of Cushing's syndrome, all corticotrophin dependent.

\section{(}


syndrome, while others seem to be mild formes frustes of the syndrome which never attain a degree of severity calling for radical therapy. We have seen two examples of the former type in the early stage of development of the syndrome. Both presented with mild increase in weight, mild hypertension, and the appearance of pale striae on the trunk. When first seen the cortisol secretion rates were 19 and $25 \mathrm{mg}$. respectively, figures not sufficiently clearly above the normal range to justify a firm diagnosis of hypercorticism.

Eighteen months later the first of these women had developed further features of Cushing's syndrome and was then found to have a secretion rate of $39 \mathrm{mg}$. daily. She subsequently underwent a subtotal adrenalectomy. The second woman was reported two years later to have developed a florid Cushing's syndrome, but no opportunity arose to determine again the cortisol secretion rate.

\section{RELATION OF CORTISOL SECRETION RATE TO URINARY 17-KETOGENIC STEROIDS}

The cortisol secretion rate is a more accurate index of adrenal cortical activity than is the urinary ketogenic steroid assay for several reasons. First, the chemistry involved in determining the specific activity of the cortisol metabolite is less complex than that involved in the analysis of unpurified urine, so that a higher analytical accuracy is readily attained. Secondly, the radioactive tracer acts as a marker and automatically takes account of any changes in the proportions of the various metabolites produced. Thirdly, the accuracy theoretically obtainable in determinations of the secretion rate is undiminished at low levels of adrenal activity. This persisting accuracy stands in sharp contrast to the weakness of most simple urinary steroid methods which become increasingly inaccurate at the lower end of the normal range of values. That the degree of correlation between 17-ketogenic steroid excretion and cortisol secretion rate is poor was suggested by Cope and Black (1959b) and further experience confirms this impression.

When the cortisol secretion rate exceeds $35 \mathrm{mg}$. daily there is usually a fair relationship between the secretion rate and the 17-ketogenic steroid excretion, the latter figure being about $50 \%$ of the former (Fig. 2). But this is not always so, and in a series of 33 cases of Cushing's syndrome all of which had cortisol secretion rates above $35 \mathrm{mg}$., the 17-ketogenic steroid excretion was found to be below $15 \mathrm{mg}$. in seven $(20 \%)$. In these cases, therefore, the results of the urine test would have been very misleading. Clinical uncertainty in the diagnosis of Cushing's

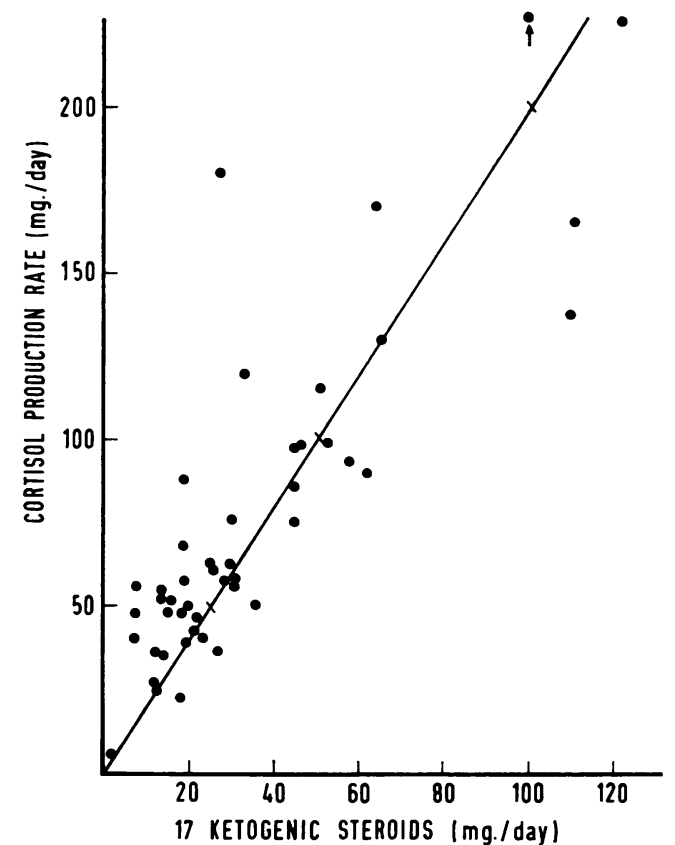

FIG. 2. Degree of correlation between cortisol secretion rate and 17-ketogenic steroid excretion in manifest hypercorticism. Line indicates excretion of $50 \%$ of secreted cortisol in the form of 17-ketogenic steroids.

syndrome is most likely to arise in cases having cortisol secretion rates between 25 and $35 \mathrm{mg}$. daily, and it is in this range that the 17-ketogenic steroid excretion assay begins to fail more seriously. In 12 such borderline cases in which the cortisol secretion lay between these limits and thus was only mildly raised, the urinary 17-ketogenic steroid assay ranged from 4 to $17 \mathrm{mg}$. In five of these cases the ketogenic steroid excretion was below $10 \mathrm{mg}$. and could have thus led to misleading conclusions (Fig. 3).

But when the ketogenic steroid excretion is clearly elevated, a raised cortisol secretion rate can confidently be deduced, provided that pregnantriol is not being excreted in excessive amounts. This complication does not usually arise in Cushing's syndrome. In 28 cases with the excretion of 17-ketogenic steroids above $20 \mathrm{mg}$. daily, the cortisol secretion rate was above $35 \mathrm{mg}$. a day in all, and was, therefore, in the Cushing's syndrome range. But a 17-ketogenic steroid excretion of less than $17.5 \mathrm{mg}$. daily does not justify the conclusion that cortisol production is also within the normal range, for among 56 subjects with such 'normal' ketogenic steroid excretion, there have been found 13 with secretion rates between 25 and $35 \mathrm{mg}$. and nine cases with secretion rates between 35 and $60 \mathrm{mg}$. 


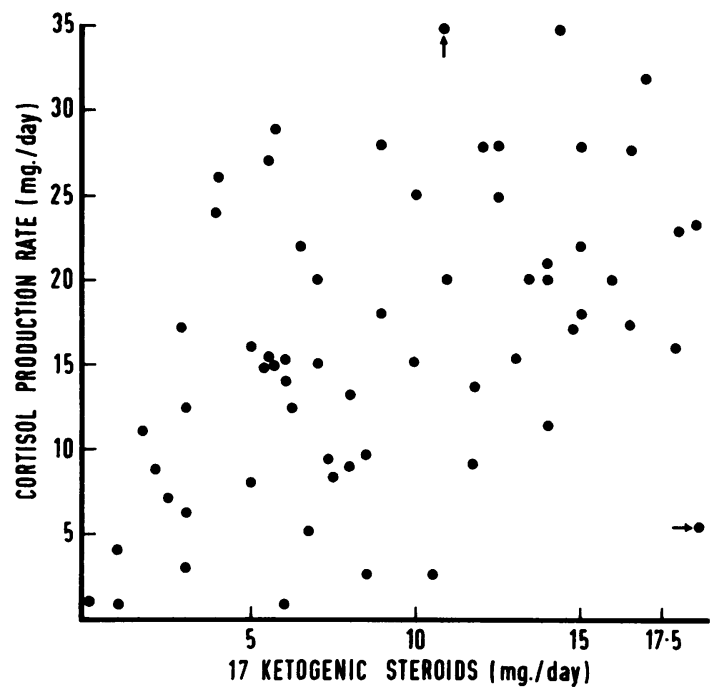

FIG. 3. Lack of correlation between cortisol secretion rate and 17-ketogenic steroid excretion in the near normal range.

daily, the latter group being cases of florid Cushing's syndrome.

In states of severe hypoadrenalism the percentage accuracy of the cortisol secretion rate estimation is not appreciably reduced, but urinary analyses of 17-ketogenic steroids become fallible at such low levels. Thus in eight patients in whom secretion rates below $5 \mathrm{mg}$. daily were recorded, the urinary 17-ketogenic steroids varied between zero and 10.5 $\mathrm{mg}$. and in four of these eight the value was $6 \mathrm{mg}$. or more. Here again the ketogenic steroid assay often brings uncertainty of interpretation.

\section{CONTINUOUS MONITORING OF CORTISOL SECRETION}

The ease of administration of the tritiated steroid and the negligible radiation hazard with which it is believed to be associated, make the day-to-day following of changes in adrenal cortisol production relatively easy and safe. The accuracy is limited mainly by the delay which inevitably occurs in the excretion of products of metabolism in the urine. When conditions are changing slowly from day to day, this will be of little significance, but when adrenal activity is rising or falling rapidly this factor may impose a delay of six to 12 hours in the apparent rate of change of activity. A further and usually smaller error arises from the fact that the products of cortisol metabolism are not all excreted in 24 hours, but that a small amount averaging 6 to $8 \%$ is delayed into the following day. With rapidly changing function this factor can lead to an error of up to $10 \%$ in the estimated activity, which h每 recently been discussed by Lazarus (1962).

In Fig. 4 are shown examples of the changes $\overrightarrow{\overline{\mathrm{H}}}$ adrenal production of cortisol under several different circumstances. Constancy of output from day day in a normal subject is shown in line $A$ which $\overline{\mathbf{S}}$ representative of several normals we have followe Line B indicates the complete lack of response t8 corticotrophin given on the second to fourth days $\mathrm{F}$ a patient with severe Addison's disease. The sensitivity of the secretion rate estimation is suct that a rise in output of even $0.5 \mathrm{mg}$. would have beegh highly significant. Curve $\mathrm{C}$ illustrates the rapt suppression of adrenal activity produced by a dose of $4 \mathrm{mg}$. of dexamethasone on the second and thired days, and curve $D$ shows a similar inhibition followed by prompt recovery of activity on stopping the steroid after the third day. Finally, curve E illus trates the rapid recovery of full cortisol production within three days after stopping long-term theray which had been maintained for 25 weeks before th test. It will be noted that the rate of recovery activity can be as rapid after long-term inhibition as it is in the two-day inhibition in curve $D$.

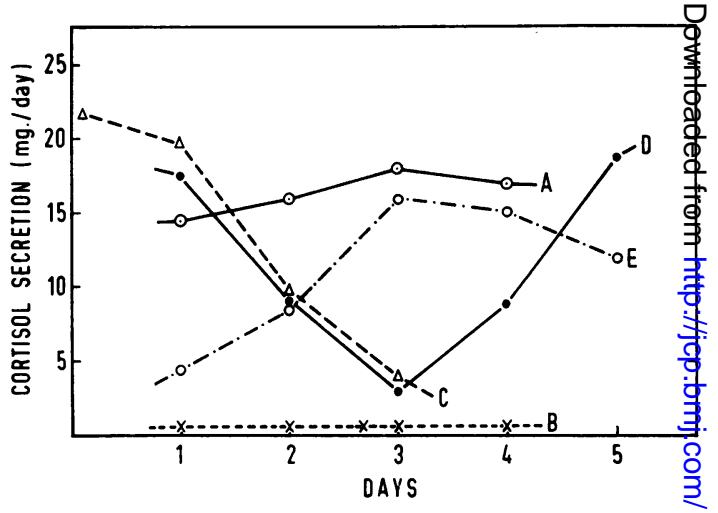

FIG. 4. Day-by-day changes in cortisol secretion rofe under varying clinical conditions described in the text.

These examples are pictured here to illustrate the ready ability of the secretion-rate technique to reveal changes in activity which are within or even well below the normal range. It is usual in our experience for such repeated secretion rate determ通ations to fall on smooth curves of the types shown here. It is evident that such techniques offer wife scope in clinical investigations.

DISCUSSION

This technique of cortisol secretion rate determ ation has now been tested and adopted by magy 
different groups, and the results of those so far published are in general confirmation of our own earlier findings (Cope and Black, 1958a and 1958b). The validity of the method has been tested directly by Lazarus (1962) who measured the 'secretion' rates in adrenalectomized subjects to whom known amounts of cortisol were administered. The observed secretion rates agreed closely with the mean of the doses given on the test and preceding days, theaverage deviation being only $5 \%$ and the largest single error $12 \%$.

Several groups have now reported series of normal cortisol secretion rates determined in this manner. Flood, Layne, Ramcharan, Rossipal, Tait, and Tait (1961) found a mean cortisol secretion of $17.2 \mathrm{mg}$. in six normal women, and Layne, Meyer, Vaishwanar, and Pincus (1962) found the mean for nine normal women to be $16.1 \mathrm{mg}$. Mlynaryk, Gillies, Murphy, and Pattee (1962) found the mean for 12 normal males to be $16.1 \pm 6.5 \mathrm{mg}$. daily. Migeon, Green, and Eckert (1963) found slightly higher values, their mean for 15 normal women being $17 \cdot 4$ mg. and for 15 normal men $20.4 \mathrm{mg}$. daily.

A mean secretion of $21.4 \mathrm{mg}$. was found in 15 normal men by Van der Straeten et al. (1963) but their method of administration of the cortisol dose by intravenous infusion prolonged over 15 minutes is one likely to raise adrenal cortical activity in many subjects.

The relatively small differences between the mean values obtained by these different groups of workers are more likely to be due to differences in clinical material than to technical differences. We believe that such a sampling difference accounts for our own recent mean value of $16.2 \pm 5.7 \mathrm{mg}$. which is appreciably higher than our original estimate of a mean normal cortisol secretion of $14.5 \mathrm{mg}$. daily, for in these two series of convalescent subjects not affected by endocrine disorders the technique was the same in each.

Since true Cushing's syndrome is essentially a state of cortisol overproduction, we have come to regard a secretion rate within normal limits as excluding the diagnosis, or at least excluding the need for major therapy. The height of the cortisol secretion rate appears to give a useful indication of the severity of the disease, and is certainly more useful for this purpose than a random single estimation of the plasma cortisol. But the decision that active therapy is needed must be based not alone on the raised secretion rate, but also on the severity and rate of progress of the patients' symptoms and of the metabolic derangements resulting. We have found only a very rough relation between the height of the cortisol secretion rate and the severity of the actual symptoms and signs.
But although we have many times encountered instances where the results of 17-ketogenic steroid excretion were in conflict with the true clinical situation as subsequently determined, this has not occurred with the cortisol secretion rate, and we have not yet had cause to lose faith in the clinical information which it provides.

The accurate measurement of low levels of adrenal activity has not, in the past, been a matter of major importance, but it is becoming so now that the induction of a medical hypophysectomy by yttrium implant is an accepted form of treatment for certain forms of metastatic carcinoma. Although there is reason to believe that the abolition of corticotrophin production is not the factor which benefits the metastatic carcinoma, yet persisting adrenal cortical function, even if this is mild, is strongly suggestive of an incomplete hypophysectomy and, if the therapeutic response has been poor, may provide a useful argument in favour of further implantation of yttrium or other seeds.

For the detection of such residual traces of adrenal activity the sensitivity of the cortisol secretion rate is ideally suited, whereas urinary assays of 17-ketogenic steroids or of 17-hydroxycorticosteroids are too insensitive at such low levels to yield sufficiently accurate information.

The cortisol secretion rate is not yet suitable for the routine screening of adrenal function, not is it desirable that it should be used for this purpose for which simpler and less specific tests are usually adequate. But in the elucidation of the difficult or ambiguous case it can produce reliable and definitive evidence of the normality or abnormality of daily cortisol production.

Our thanks are due to Miss Margaret Harwood for technical help in these studies, and to the Medical Research Council who provided a grant for her. We are grateful also to colleagues for allowing us access to some of the cases, and to the U.S. National Institutes of Health for generous samples of ${ }^{14} \mathrm{C}$-cortisol used in the earlier years.

\section{REFERENCES}

Bush, I. E. (1952). Biochem. J., 50, 370.

Cope, C. L., and Black, E. G. (1958a). Clin. Sci., 17, 147.

- - - (1958b). Brit. med. J., 1, 1020.

- - (1959a). J. Obstet. Gynaec. Brit. Cwlth, 66, 404.

- (1959b). Brit. med. J., 2, 1117.

_ and Pearson, J. (1963). Clin. Sci., 25, 331.

Eberlein, W. R., and Bongiovanni, A. M. (1955). Arch. Biochem., 59, 90.

Flood, C., Layne, D. S., Ramcharan, S., Rossipal, E., Tait, J. F., and Tait, S. A. S. (1961). Acta endocr., (Kbh.), 36, 237.

Layne, D. S., Meyer, C. J., Vaishwanar, P. S., and Pincus, G. (1962). J. clin. Endocr., 22, 107.

Lazarus, L. (1962). Ibid., 22, 581.

Migeon, C. J., Green, O. C., and Eckert, J. P. (1963). Metabolism, 12, 718.

Mlynaryk, P., Gillies, R. R., Murphy, B., and Pattee, C. J. (1962). J. clin. Endocr., 22, 587.

Van der Straeten, M., Vermeulen, A., and Orie, N. (1963). Acta endocr. (Kbh.), 43, 430. 